\title{
Magnetic helicity ejections and coronal activity
}

\author{
A. Nindos $^{1}$ \\ ${ }^{1}$ Section of Astrogeophysics, Physics Department, University of Ioannina, \\ Ioannina GR-45110, Greece \\ email: anindos@cc.uoi.gr
}

\begin{abstract}
Magnetic helicity quantifies the degree of linkage and/or twistedness in the magnetic field. It is probably the only physical quantity which is approximately conserved even in resistive MHD. This makes it an ideal tool for the exploration of the physics of solar eruptions. In this article, I discuss the sources of magnetic helicity injected into active regions and I point out that coronal mass ejections (CMEs) are probably necessary to remove at least part of the excess helicity produced in the Sun. I also discuss the importance of magnetic helicity in the overall coronal evolution that may lead to eruptions.
\end{abstract}

Keywords. Sun: activity, Sun: magnetic fields, Sun: coronal mass ejections (CMEs), Sun: flares

\section{Introduction}

Magnetic helicity, $H$, quantifies the degree of linkage and/or twistedness in the magnetic field, and therefore provides a measure of the topological complexity of the field. The "natural" unit of magnetic helicity is the square of magnetic flux $\left(\mathrm{Mx}^{2}\right)$ and therefore, in the simplest case the helicity of an isolated uniformly twisted flux tube with $N$ turns and magnetic flux equal to $\Phi$ is simply $N \Phi^{2}$.

It is well established (e.g. see Berger 1984) that magnetic helicity is very well preserved in plasmas with high magnetic Reynolds numbers, even in the presence of dissipative processes such as magnetic reconnection (more accurately, it is approximately conserved on time scales smaller than the global diffusion time scale; see Berger 1984). This property of helicity has important consequences in the evolution of magnetic fields: a stressed magnetic field cannot relax to a potential field. This behavior may have important implications for the initiation of flares and coronal mass ejections (CMEs). On the other hand, our inability to measure solar magnetic fields accurately above the photosphere makes helicity calculations difficult because they require knowledge of either the three-dimensional magnetic field in all or part of the coronal volume, including its lower boundary (e.g. Berger 1984; Finn and Antonsen 1985) or the flow velocities on this lower boundary (e.g. Berger and Field 1984).

In this article, I present a short review of magnetic helicity of active regions (ARs) with emphasis on its role in the initiation of solar eruptions. The article is organized as follows. After defining magnetic helicity and its flux, in section 3 I outline the methods for its computation using solar data. In section 4, I discuss the sources of magnetic helicity in ARs and in section 5 I examine its role in eruptive phenomena. Conclusions are presented in section 6 . For more extensive reviews of the subject, the reader is referred to the articles by Démoulin (2007) and Démoulin \& Pariat (2009). 


\section{Definitions}

\subsection{Magnetic helicity}

For a magnetic field $\mathbf{B}$ fully contained within a volume $V$ (i.e. at any point of its boundary $S$ the normal component $B_{n}=\mathbf{B} \cdot \hat{\mathbf{n}}$ vanishes), magnetic helicity is defined as

$$
H=\int_{V} \mathbf{A} \cdot \mathbf{B} \mathrm{d} V
$$

where $\mathbf{A}$ is the magnetic vector potential $(\mathbf{B}=\nabla \times \mathbf{A}) . H$ is independent of the gauge selection for $\mathbf{A}$ (i.e. independent of the transformation $\mathbf{A} \rightarrow \mathbf{A}+\nabla \Phi$, where $\Phi$ is any single-valued derivable function of space and time).

In the solar atmosphere magnetic flux passes through $S$ (especially in the photosphere) and therefore the above condition is not satisfied. However, Berger \& Field (1984) and Finn \& Antonsen (1985) have shown that when $B_{n} \neq 0$ on $S$, we can define a gaugeinvariant relative magnetic helicity (hereafter refered to as helicity) of $\mathbf{B}$ with respect to the magnetic helicity of a reference field $\mathbf{B}_{p}$ having the same distribution of normal magnetic flux on the surface $S$ surrounding $V$ :

$$
H=\int_{V} \mathbf{A} \cdot \mathbf{B} \mathrm{d} V-\int_{V} \mathbf{A}_{p} \cdot \mathbf{B}_{p} \mathrm{~d} V
$$

where $\mathbf{A}_{p}$ is the vector potential of $\mathbf{B}_{p}$. The quantity $H$ does not depend on the common extension of $\mathbf{B}$ and $\mathbf{B}_{p}$ outside $V$. Being a potential field it is a convinient choice for $\mathbf{B}_{p}$. If in addition $\nabla \cdot \mathbf{A}_{p}=0$ and $\left(A_{p}\right)_{n}=0$ on $S$ then the term $\int_{V} \mathbf{A}_{p} \cdot \mathbf{B}_{p} \mathrm{~d} V$ vanishes (Berger 1988), so $H$ has the same expression as in the case of the helicity in closed volumes (eq. 2.1).

\subsection{Flux of magnetic helicity}

Generally, the amount of helicity within $V$ can change either due to helicity flux crossing $S$ or/and due to dissipation within $V$. Berger (1984) has demonstrated that the helicity dissipation rate is negligible in all processes taking place in the corona, including reconnection and all non-ideal processes. Helicity's dissipation time scale is the global diffusion time scale and consequently it can be regarded as an almost conserved quantity even in resistive MHD.

In the solar atmosphere $V$ is part of the coronal volume, bounded from below by a portion of the photosphere $S_{p}$ and bounded in the corona by $S_{c}\left(S_{c}=S-S_{p}\right)$. No data can presently provide $\mathbf{B}$ on any $S_{c}$ surface. The helicity flux across $S_{c}$ can only be estimated indirectly by the helicity carried away by CMEs, and estimated in the interplanetary space from the associated magnetic clouds. All studies compute the helicity injected at the photospheric level through $S_{p}$. Using the gauge $\nabla \cdot \mathbf{A}_{p}=0$, and selecting the boundary condition $\mathbf{A}_{p} \cdot \hat{\mathbf{n}}=0$ for the vector potential of the potential reference field, Berger \& Field (1984) derived the flux of magnetic helicity through a planar surface:

$$
\frac{d H}{d t}=2 \int_{S_{p}}\left[\left(\mathbf{A}_{p} \cdot \mathbf{B}_{t}\right) v_{n}-\left(\mathbf{A}_{p} \cdot \mathbf{v}_{t}\right) B_{n}\right] \mathrm{d} S
$$

where $B_{t}$ and $B_{n}$ are the tangential and normal components of the photospheric magnetic field and $v_{t}$ and $v_{n}$ the tangential and normal compoments of the photospheric plasma velocity. 


\section{Computations of helicity in finite volumes}

\subsection{Computations of instantaneous helicity in the corona}

The application of the concept of relative magnetic helicity defined in section 2 is problematic in AR magnetic field extrapolations because the volumes involved are finite. For linear force-free (LFF) fields, this problem can be bypassed by formulating the helicity content of an AR in terms of surface integrals applied to the lower boundary (Berger 1985, Georgoulis and LaBonte 2007). These calculations include summations over the Fourier modes of the magnetogram. When the force-free parameter $\alpha$ exceeds a certain critical value that depends on the horizontal size of the calculation box, helicity attains unphysical large values, and Démoulin et al. (2002b) proposed to use the linearized Berger's (1985) expression in which the helicity is proportional to $\alpha$. In several publications, (e.g. Démoulin et al. 2002b; Green et al. 2002; Nindos and Andrews 2004) this linearized formula has been used in conjunction with the best value of $\alpha, \alpha_{b e s t}$, which is determined by comparing the computed LFF field lines with the observed soft X-ray or EUV coronal structures.

Georgoulis et al. (2012) extended the work by Georgoulis and LaBonte (2007) on LFF fields to non-linear force-free (NLFF) fields. They developed a method that depends on a lower-boundary connectivity matrix that can be inferred either by an NLFF field extrapolation or otherwise. Instead of using extrapolation results, these authors used a unique connectivity-matrix solution for a given flux-partition map. This solution relies on a simulated annealing algorithm designed to minimize the distances of connected opposite polarity partitions.

In several publications that treat the general problem of the calculation of the instantaneous helicity in the corona, NLFF field extrapolations are used, and the helicity in the computation box is estimated using methods that are based on the Coulomb gauge (e.g. Rudenko and Myshyakov 2011; Thalmann et al. 2011). The choice of gauge is irrelevant for the relative magnetic helicity value, but it may influence how computationally expensive the algorithm becomes. Valori et al. (2012) exploited the gauge freedom by choosing one that requires that one component of the vector potential vanishes; their method is a direct extension of an earlier work by DeVore (2000) to finite volumes.

\subsection{Computations of the injection rate of helicity}

When high-cadence photospheric magnetograms are available, the horizontal velocity appearing in eq. (2.3) can be computed using various techniques. In the first studies of the helicity injection rate, several authors (e.g. Chae 2001; Nindos \& Zhang 2002; Moon et al. 2002a,b; Nindos et al. 2003; Chae et al. 2004) utilized the local correlation tracking (LCT) technique (November and Simon 1988) for the computation of horizontal velocities. Démoulin \& Berger (2003) have pointed out that with magnetograms one follows the photospheric intersection of the magnetic flux tubes but not the evolution of the plasma. Consequently, from the observed magnetic evolution we obtain the flux tube motion and not the plasma motion parallel to the photosphere. If $\mathbf{v}_{t}$ is the tangential component of the photospheric plasma velocity and $v_{n}$ the velocity perpendicular to the photosphere, the tracking algorithm detects the velocity of the footpoints of the flux tube which is

$$
\mathbf{u}=\mathbf{v}_{t}-\frac{v_{n}}{B_{n}} \mathbf{B}_{t}
$$

However, MHD simulations (e.g. Welsch et al. 2007) have shown that this formula is not valid always, and its use should be treated with caution especially during flux emergence episodes. 
The combination of eq. (3.1) and (2.3) shows that the whole helicity flux density can be retrieved within the accuracy of the calculation. Consequently, one may use the quantity $G_{A}=-2 \mathbf{u} \cdot \mathbf{A}_{p} B_{n}$ as a proxy to the whole helicity flux density.

$G_{A}$ has been used extensively in several studies (e.g., see the references for the LCT usage). The $G_{A}$ maps always appear extremely complex both in space and time, with polarities of both signs present at any time. Pariat et al. (2005) showed that $G_{A}$ is not a real helicity flux density and that its properties introduce artificial polarities of both signs. The spurious signals appear due to the fact that helicity flux densities per unit surface are not physical quantities. Due to the properties of helicity, only helicity flux density per unit of elementary magnetic flux has a physical meaning. But to estimate such quantity using real observations, it is necessary to isolate flux tubes and determine their connectivity, which is actually not possible. Thus any definition of a helicity flux density will only be a proxy of the helicity flux density per unit magnetic flux. Pariat et al. (2005) introduced a new proxy for helicity flux density, $G_{\theta}$, which does not suffer from $G_{A}$ 's problems. $G_{\theta}$ implies that the helicity injection rate is the summation of the rotation rate of all pairs of elementary fluxes weighted by their magnetic flux. An example of a $G_{\theta}$ map is given in fig. 1 .

In order to define the real helicity flux density, the coronal linkage needs to be provided. With it one can represent how all elementary flux tubes move relatively to a given elementary flux tube, and the helicity flux density is defined per elementary flux tube. Using photospheric maps this can be achieved by distributing equally the helicity input between the two footpoints for each elementary flux tube. Then the helicity flux can be rewritten as a flux of magnetic helicity per unit of surface, $G_{\Phi} . G_{\Phi}$ is a field-weighted average of $G_{\theta}$ at both photospheric footpoints, $\mathbf{x}_{ \pm}$, of the photosheric connection.

While $G_{\Phi}$ provides the true helicity flux density, its practical use is presently limited by our ability to define the coronal linkage for all magnetic polarities. Currently, all we can

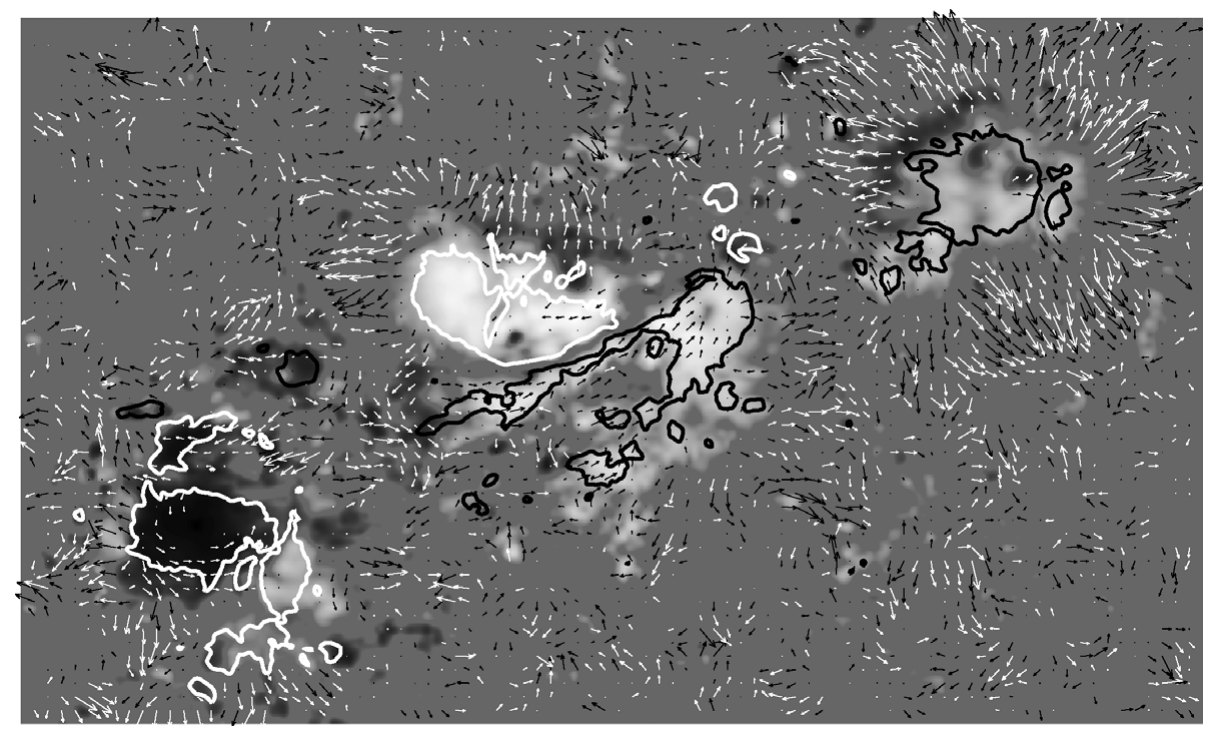

Figure 1. Computed velocity vectors and the corresponding $G_{\theta}$ map (gray-scale image) for AR11158 on February 152011 01:15 UT. The velocities have been computed using the DAVE method (Schuck 2006). The maximum arrow length measures velocity of $1 \mathrm{~km} \mathrm{~s}^{-1}$. The black and white thick contours represent longitudinal magnetic field strengths of 700 and $-700 \mathrm{G}$, respectively. 
do is to estimate $G_{\Phi}$ maps for models that resemble certain configurations and evolution patterns (Pariat et al. 2006).

It has been reported (e.g. Pariat et al. 2006; 2007) that unlike the usual $G_{A}$ maps, most $G_{\theta}$ maps show almost unipolar spatial structures because the nondominant helicity flux densities are significantly suppressed. However, as can be seen from fig. 1, this is not always the case. If a crude modeling of the expected $G_{\Phi}$ morphology shows that $G_{\Phi}$ should be unipolar then the bipolar structures of the computed $G_{\theta}$ maps should be spurious. Note that, according to our modeling, this was not the case in the mixed polarity morphology of the $G_{\theta}$ map of fig. 1 .

When a cube of $G_{A}$ or $G_{\theta}$ maps is available, one can calculate the time evolution of the total helicity flux, $d H / d t$. An example using a time series of $G_{\theta}$ maps which includes the $G_{\theta}$ map of fig. 1, appears in fig. 2 (top panel). The resulting time profile of the net accumulated change of helicity shows in the middle panel of fig. 2 . Note that we expect that the helicity flux integrated using $G_{A}$ to be identical to the one integrated using $G_{\theta}$ because both definitions are derived from eq. (2.3). The interested reader may refer to Pariat et al. (2006) for a discussion on the small differences that appear in practical computations.

\section{Sources of magnetic helicity}

The first term of the right-hand side of eq. (2.3) corresponds to the injection of helicity by advection (i.e. emergence of field lines that cross the photosphere) while the second

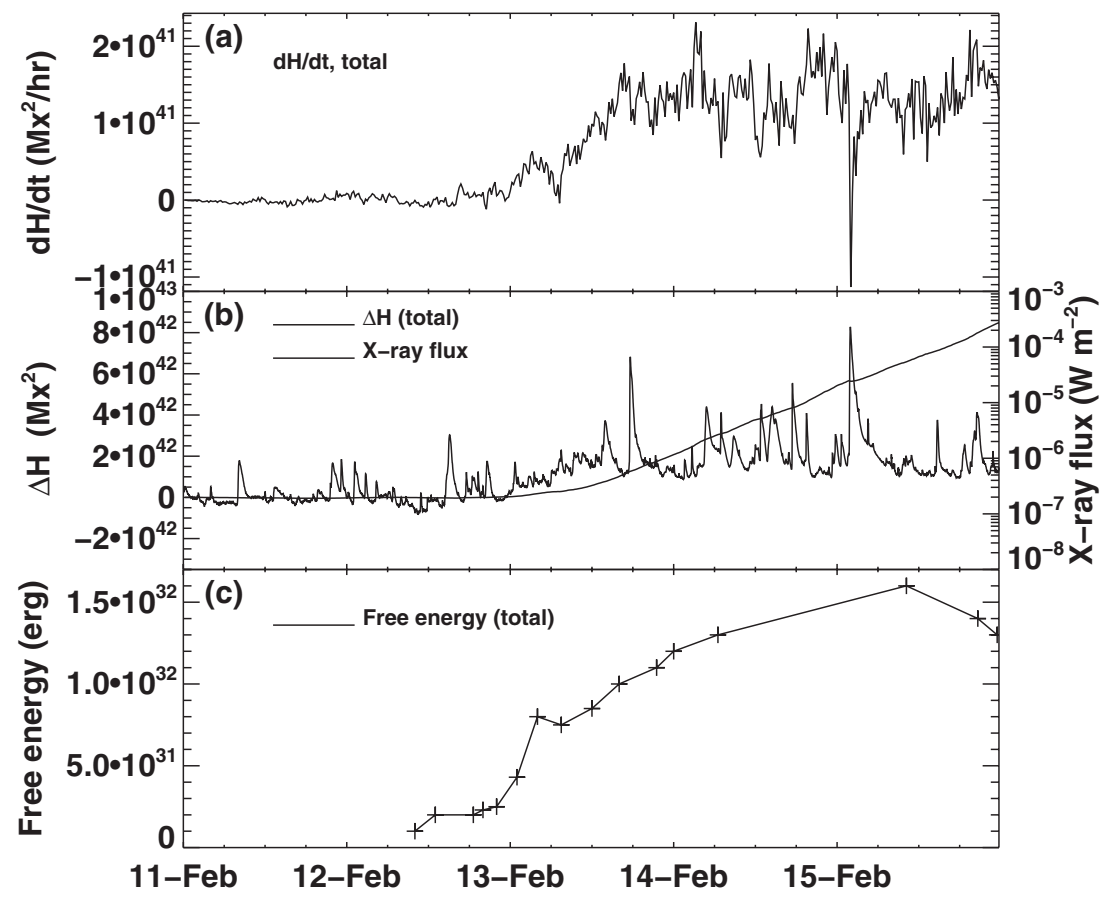

Figure 2. (a) Time profile of the net flux of injected helicity, $d H / d t$, over the field of view of AR11158 that is presented in fig. 1. (b) Time profile of the net accumulated change of helicity, $\Delta H$ in AR11158, calculated from the measured $d H / d t$. The GOES soft X-ray flux time profile is also presented. (c) Temproral evolution of the magnetic free energy for AR11158 (modified from Nindos et al. 2012). 
term (also known as shearing term) is the flux of helicity due to motions parallel to $S$. Such motions may come either from differential rotation and/or transient photospheric shearing flows.

Differential rotation was the first mechanism that injects helicity into ARs which was studied (DeVore 2000). Even when a single bipole is considered, differential rotation does not provide a monotonous input of magnetic helicity (DeVore 2000). This is because differential rotation rotates both magnetic polarities on themselves and also changes their relative positions, introducing twist and writhe helicity fluxes, respectively. These fluxes always have opposite signs and similar amplitudes, and therefore partially cancel (Démoulin et al. 2002a). Démoulin et al. (2002b) and Green et al. (2002) studied the long-term evolution of the helicity injected by differential rotation into the coronal part of two active regions which were followed from their birth until they decayed. These studies showed that the contribution of differential rotation to the helicity budget of active regions is small.

The study of the helicity budget of active regions requires knowledge of the helicity injected into them and of the helicity carried away from them. The former is computed using the methods described in section 3 while CMEs are considered responsible for the latter. The helicity content of a CME can be estimated by the change of coronal helicity of the source region during the event (e.g. Mandrini et al. 2005). Inside magnetic clouds, helicity is estimated from modeling of the in situ measurements of the magnetic field vector. In practice, in studies of the long-term evolution of helicity of active regions that are linked to at least one magnetic cloud at $1 \mathrm{AU}$ one assumes that the helicity carried away by each CME is equal to the helicity content in the magnetic cloud. Nindos et al. (2003) and Lim et al. (2007) were able to partially reconcile the amount of helicity injected into the corona with the helicity carried away by the CMEs in the active regions they studied. However, the uncertainties of these studies are significant primarily due to the large uncertainties in the calculation of the helicity transported away by CMEs.

Using line-of-sight magnetograms together with the Démoulin and Berger (2003) formula (eq. 3.1), one cannot calculate separately the advection and the shearing term in eq. (2.3). Furthermore, the validity of the Démoulin and Berger hypothesis has been questioned (e.g. Schuck 2008; Ravindra et al. 2008; Liu and Schuck 2012; see also section 3.2).

The separate computation of both the shearing and advection terms requires the use of photospheric vector magetograms. Several methods have been developed towards this goal (e.g. Kusano et al. 2002; Welsch et al. 2004; Longcope 2004; Georgoulis \& LaBonte 2005; Schuck 2008; Zhang et al. 2012; Liu \& Schuck 2012). Most of these methods have not been tested extensively with solar data, and contradictory results have been reported. Furthermore, the methods which were developed before 2007 were checked against the same anelastic MHD simulation (Welsch et al. 2007) and produced different results.

\section{Magnetic helicity and the initiation of CMEs}

\subsection{Helicity as an important agent for CME initiation}

In several publications, CMEs are thought to be the primary agent through which the Sun gets rid of its excess helicity (e.g. Rust 1994; Low 1996; Zhang and Low 2005). The main arguments for this conclusion is that: (1) on the global scale, helicity emerges predominantly negative in the northern hemisphere and predominantly positive in the southern hemisphere (e.g. Pevtsov et al. 1995), and this hemispheric helicity sign pattern does not change from solar cycle to solar cycle (Pevtsov et al. 2001). (2) A fraction of 
AR's helicity is cretaed by the dynamo and then transported into the corona through the photosphere with the emerging magnetic flux. This process would constantly accumulate helicity into the corona because of helicity's property not to be destroyed under reconnection. Furthermore, cancellations of opposite helicity fluxes involves a small fraction of the magnetic flux (e.g. between ARs of opposite helicity sign, either within the same hemisphere or across the equator, Pevtsov 2000). Finally, it is speculated that a small fraction of helicity is lost from the corona by the reconnections between ARs and coronal holes, since the relevant magnetic fluxes are small.

Along the above lines, Low and Zhang (2002) and Zhang and Low (2001; 2003) have developed a unified view of CMEs as the last chain of processes that transfer helicity from the convective zone into the inteplanetary medium. Their theory exploits Taylor's conjecture that the magnetic field will relax towards a LFF field state. A summary of their results is as follows. When new field enters the corona repeated reconnections between the new and pre-existing field take place. This process simplifies the magnetic topology and the dissipated magnetic energy produces flares. The relaxation proceeds according to Taylor's conjecture and results in the formation of a flux rope which contains a significant fraction of the total helicity of the system. The fate of the flux rope is determined by the efficiency of its confinement by its surrounding anchored field. Flux rope ejection occurs when the magnetic energy it contains is sufficient to drive an outward expansion against the confining field.

In a series of articles Zhang et al. (2006; 2008; 2012) studied theoretically the maximum amount of helicity that can be stored in various field configurations and its implications for coronal evolution. In the 2006 article they showed that in an open spherical volume like the corona, for a given boundary flux distribution, there is an upper bound on the magnitude of the total helicity of all axisymmetric power-law force-free fields. When the accumulated helicity exceeds this limit, a non-equilibrium situation is reached which mimics the initiation of CMEs. In the 2008 article they found that the helicity upper bound of force-free fields depends on the boundary flux distribution: multipolar photospheric configurations can have a helicity upper bound 10 times smaller than dipolar ones. In the 2012 article they studied the helicity of self-similar axisymmetric force-free fields and found that there may be an upper bound on the total helicity of all bipolar axisymmetric force-free fields. As the helicity increases, the fields open up forming a current sheet surrounded by Parker-spiral-like structures.

Amari et al. (2003a, 2003b) constructed a set of force-free fields having different magnetic flux and helicity contents and used them as initial conditions by applying converging motions or a diffuse-driven evolution. These processes can trigger eruptive events that may be either confined or global, depending on the value of the initial helicity. Amari et al. (2003b) concluded that helicity cannot be the only parameter controlling the triggering of an ejection: having a large enough helicity seems a necessary condition for an ejection to occur, but not a sufficient one. Jacobs et al. (2006) performed MHD simulations by shearing an axisymmetric arcade outside a sphere, and found that a twisted flux tube forms and ejects when $H / \Phi^{2}$ is typically above 0.2-0.3.

\subsection{Observational evidence}

The physical view presented in section 5.1 has been supported by several observations. Nindos and Andrews (2004) used LFF field extrapolations and the $\alpha_{b e s t}$ method to model the pre-flare coronal field of 78 ARs that produced big flares. Only some $60 \%$ of these flares were associated with CMEs. Then from the derived values of $\alpha_{b e s t}$ they computed the corresponding coronal helicities. Their results indicated that in a statistical sense both the pre-flare absolute value of $\alpha$ and the corresponding coronal helicity of the ARs 
producing CME-associated big flares were larger than the absolute value of $\alpha$ and helicity of those that did not have associated CMEs.

The above results are consistent with the ones reported by LaBonte et al. (2007) who calculated the helicity flux in 48 ARs that produced X-class flares and in 345 non-Xflaring ARs. They found that a necessary condition for the occurrence of an X-class flare is that the peak helicity flux has a magnitude $>6 \times 10^{36} \mathrm{Mx}^{2} \mathrm{~s}^{-1}$.

I have also studied the helicity evolution of 43 ARs that emerged on the solar disk within $45^{\circ}$ from disk center. The database consisted of 18 ARs that gave a CME before crossing $\mathrm{W} 45^{\circ}$ and 25 that did not. The helicity calculations for each AR were performed from the time of its emergence until the time of its first CME or the time when it reached $\mathrm{W} 45^{\circ}$ (whichever happened first). At a given time $t$, the coronal helicity $H_{c}$ was estimated by the formula $H_{c}(t)=H_{c, \text { emerg }}+\Delta H_{i n j}(t)$ where $H_{c, \text { emerg }}$ was the helicity stored in the corona at the beginning of flux emergence and $\Delta H_{i n j}(t)$ was the accumulated change of helicity as a function of time. The quantity $H_{c, \text { emerg }}$ was calculated using LFF field extrapolations and the $\alpha_{\text {best }}$ method; this procedure was reliable because the field in most cases emerged in a nearly potential state. The quantity $\Delta H_{i n j}$ was calculated from the helicity fluxes $d H / d t$ using the method described in section 3.2.

The results appear in fig. 3 (left column) and indicate that in a statistical sense the coronal helicity of the ARs that produced a CME before crossing W45 $5^{\circ}$ was larger than the coronal helicity of those that crossed $\mathrm{W} 45^{\circ}$ without producing a CME. The right column of fig. 3 shows the coronal helicities at two different times in the evolution of the ARs: the bottom panel shows the coronal helicities when the ARs produced their first confined flares above C1.0 while the top panel shows the coronal helicities when the ARs
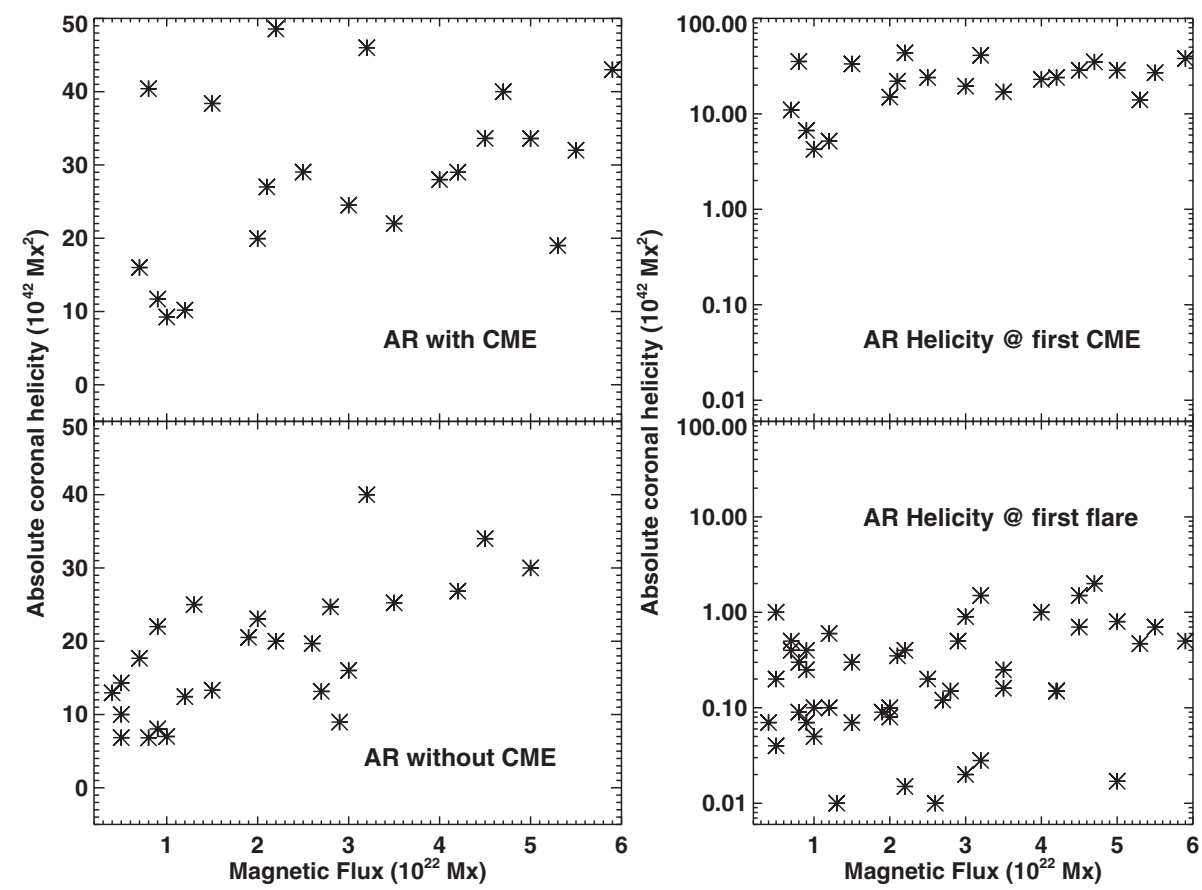

Figure 3. Left column, top: scatter plot of coronal helicities of emerging ARs just before their first CMEs as a function of the AR's magnetic fluxes. Left column, bottom: same as top panel, but for ARs when they cross $\mathrm{W} 45^{\circ}$ without producing any CME. Right column, top: same as left column, top. Right column, bottom: same as top panel, with the exception that the helicities were calculated just before the first confined flare of each AR (see text for details). 
produced their first CMEs. The segregation of the two sets of helicity values is almost complete. This result implies that, unlike CMEs, confined flares may occur without the prior accumulation of significant amounts of helicity.

Tziotziou et al. (2012) used the method developed by Georgoulis et al. (2012; see section 3.1) to calculate the instantaneous magnetic free energy and helicity of 162 vector magnetograms that corresponded to 42 different ARs. They found a statistically significant, monotonic correlation between the free energy and helicity. This correlation implies that, in addition to free energy, helicity may play a significant role in eruptive phenomena. In their study, the eruptive ARs appeared well segregated from the non-eruptive ones in both free energy and helicity (see fig. 4).

In ARs, a primary constraining force that inhibits global eruptions is provided by the overlying background field. Using both line-of-sight and vector magnetograms, Nindos et al. (2012) studied the long-term evolution of the background field in AR11158 that produced three major CMEs. In their calculations they used the decay index of the magnetic field which is a parameter that quantifies how fast the field decreases with height. Their results indicated that the initiation of eruptions did not depend critically on the temporal evolution of the variation of the background field with height. On the other hand, they showed that both the magnetic free energy (computed from NLFF field extrapolations) and the accumulated helicity into the corona (computed using the method described in section 3.2) contributed the most to the eruptions by their increase throughout the observations (by factors of more than 5 and more than two orders of magnitude, respectively; see fig. 2).

\subsection{Other approaches}

There are several other approaches to the initiation of CMEs and the role of helicity. Some models suggest that eruptive events can occur without any significant helicity accumulations. MacNeice et al. (2004) studied the evolution of helicity under the breakout model (Antiochos et al. 1999). In their simulation, the model was driven by a shear flow

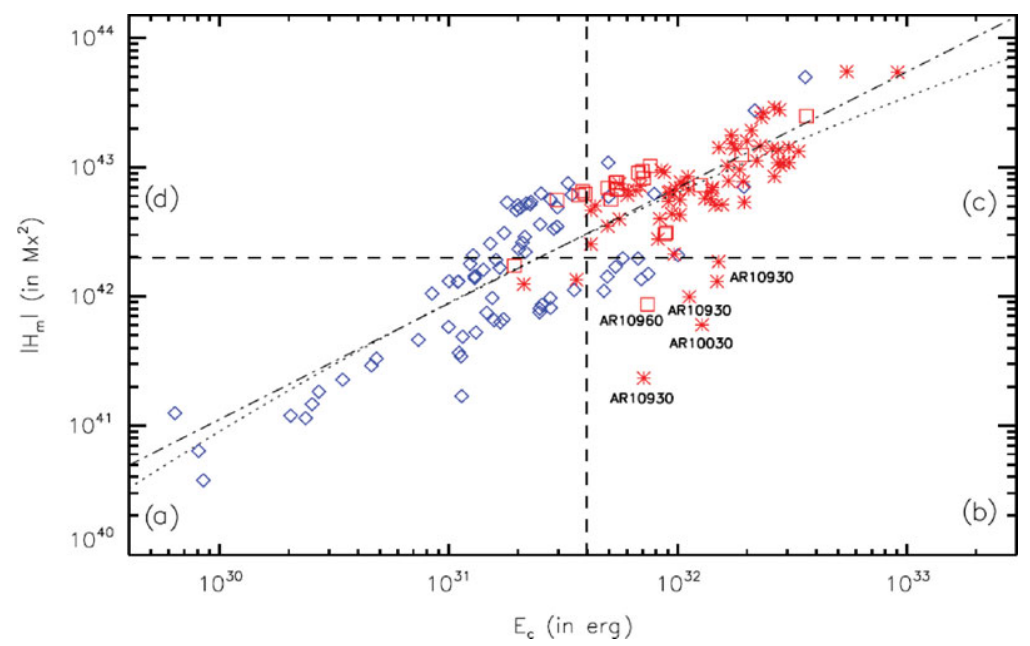

Figure 4. Free energy-helicity diagram of ARs. Diamonds, squares and asterisks correspond to non-flaring, M- and X-class flaring ARs, respectively. Dashed lines indicate the estimated thresholds for helicity and magnetic free energy above which ARs give major flares. These thresholds divide the diagram into four regions, labeled a, b, c, and $\mathrm{d}$. The dotted and dashed-dotted lines denote the least-squares best fit and the least-squares best logarithmic fit, respectively, between helicity and magnetic free energy (from Tziotziou et al. 2012). 
that injected both free energy and net helicity into the corona. Their results showed that the helicity shed by the plasmoid ejection was at least $80 \%$ of the total originally injected into the system. They interpreted this result as an indication that although CMEs remove the bulk of the coronal helicity, some fraction remains behind. They suggested that some other mechanism (possibly small-scale diffusion) might be responsible for dissipating the rest of the helicity. Furthermore, Kliem et al. (2011) found that simulated flux rope CMEs carried away only a minor part of the initial helicity that was present in the simulation box; most of the helicity remained in the simulation box even after the departure of the CME from the simulation box. This result was interpreted as a consequence of the requirement that the current through an expanding loop must decrease if the magnetic energy of the configuration is to decrease as the loop rises, to provide the kinetic energy of the CME.

Phillips et al. (2005) presented simulations of the breakout model where eruption occurs even when no net helicity is injected into the corona. In their simulations the eruption occurs at a fixed magnitude of free energy in the corona, independent of the value of helicity. It would be desirable to check these results against computations of the helicity evolution in observed eruptions that appear to be due to breakout. The MHD simulations by Zuccarello et al. (2009) also showed that the injection of helicity is not a necassary constraint in the initiation of CMEs. However, the absence of significant net helicity accumulation prior to an eruption might result from the accumulation of similar amounts of both positive and negative helicity; in such case the "helicity anihilation" might be at work (Kusano et al. 2003). Indeed, Kusano et al. (2004) presented simulations where the introduction of a reverse helicity was essential for the eruption of a sheared arcade.

Moreover, several proposed eruption mechanisms do not explicitly rely on helicity. These models include the tether cutting (e.g. Moore et al. 2001); breakout (Antiochos et al. 1999); magnetic flux cancellation (e.g. van Ballegooijen and Martens 1989); and the torus instability (Kliem and Török 2006). On the other hand, helicity is at the heart of the helical kink instability (e.g. Rust and Kumar 1996; Török and Kliem 2005; Kliem et al. 2012) which has been invoked to explain several eruptive phenomena.

\section{Conclusions}

Magnetic helicity provides an important tool for the study of eruptive phenomena. Its use is justified by two key properties of CMEs: (1) the pre-eruption magnetic topology is non-potential, and (2) CMEs carry away twisted magnetic fields. Of course, there are also other physical quantities that describe non-potential fields, for example the $\alpha$ parameter of the LFF field approximation. But helicity is superior because of its unique feature of being conserved even in resistive MHD on time scales less than the global diffusion time scale. This makes helicity probably the only physical quantity which can monitor the entire history of an eruptive event: from the transfer of magnetic field from the convective zone all the way to the eruption and the escape of the CME into interplanetary medium. On the other hand, calculations of helicity are difficult and only relatively recently attempts have been made to measure helicity using solar observations.

A lot of effort has been put on the determination of the relative importance of the sources of the helicity that is injected into ARs. Theoretical and observational work has demonstrated that differential rotation is an inefficient mechanism to account for the helicity budget of ARs. Computations using high-cadence longitudinal magnetograms give the total helicity flux but cannot separate the shearing from the advection term. Attempts for the computation of the shearing and advection term separately have been made using vector magnetograms. But the algorithms that have been developed have not 
been applied extensively to observations. Even more serious uncertainties are associated with the computation of the helicity carried away by CMEs. All the above problems contribute to the discrepancies concerning the helicity budget of ARs. At this point, these uncertainties have been cleared up only partially and much work needs to be done on this issue.

There is concensus that CMEs are probably necessary to remove at least part of the excess helicity produced in the Sun. As for the role of helicity in the initiation of eruptions, theoretical studies indicate that eruptions can occur with or without the prior accumulation of significant amounts of helicity into the ARs that erupt. The analysis of a few observational data sets has indicated that helicity accumulation might be a necessary but not sufficient condition for an eruption to occur. This result requires confirmation with the analysis of more data, both in a statistical sense and in case studies. It appears that for a thorough investigation of the magnetic origin of eruptions other parameters might be also important, for example the location with respect to the pre-existing field where helicity is injected, the efficiency of the reconnection process and how efficiently the helicity-charged stucture is confined by the overlying magnetic field.

To make progress in the subject of the evaluation of the importance of helicity in the initiation of eruptions we need to: (1) improve the reliability and accuracy of the helicity computations, (2) compare the helicity budgets between non-eruptive and eruptive ARs in the pre-eruption state, and (3) link observations with an eruption initiation mechanism that will be able to capture most of the observed features of the eruption.

\section{Acknowledgments}

I thank the organizers of IAU Symposium 294 for the financial support. This research has been partly co-financed by the European Union (European Social Fund -ESF) and Greek national funds through the Operational Program "Education and Lifelong Learning" of the National Strategic Reference Framework (NSRF) -Research Funding Program: "Thales. Investing in knowledge society through the European Social Fund"

\section{References}

Antiochos, S. K., DeVore, C. R., \& Klimchuk, J. A. 1999, ApJ, 510, 485

Amari, T., Luciani, J. F., Aly, J. J., Mikic, Z., \& Linker, J. 2003a, ApJ, 585, 1073

Amari, T., Luciani, J. F., Aly, J. J., Mikic, Z., \& Linker, J. 2003b, ApJ, 595, 1231

Berger M. A. 1984 Geophys. Astrophys. Fluid Dyn., 30, 79

Berger M. A. 1985 ApJS, 59, 433

Berger M. A. 1988 A\&A, 201, 355

Berger, M. A., \& Field, G. B. 1984 J. Fluid Mech., 147, 133

Chae, J. 2001 ApJ (Letters), 560, L95

Chae, J. 2007 Adv. Sp. Res., 39, 1700

Chae, J., Moon, Y.-J., \& Park, Y.-D. 2004 Solar Phys., 223, 39

Démoulin, P. 2007 Adv. Sp. Res., 39, 1674

Démoulin, P. \& Berger, M. A. 2003 Solar Phys. 215, 203

Démoulin, P. \& Pariat, E. 2009 Adv. Sp. Res., 43, 1013

Démoulin, P., Mandrini, C. H., van Driel-Gesztelyi, L., Lopez-Fuentes, M.C, \& Aulanier, G. 2002a Solar Phys. 207, 87

Démoulin, P., Mandrini, C. H., van Driel-Gesztelyi, L., et al. 2002b AESA, 382, 650

DeVore, R. C. 2000 ApJ, 539, 944

Finn, J. H., \& Antonsen, T. M. J. 1985 Comments Plasma Phys. Contr. Fus., 9, 111

Georgoulis, M. K., \& LaBonte, B. J. 2006 ApJ, 636, 475 
Georgoulis, M. K., \& LaBonte, B. J. 2007 ApJ, 671, 1034

Georgoulis, M. K., Tziotziou, K., \& Raouafi, N. 2012 ApJ, 759, 1

Green, L. M., Lopez-Fuentes, M. C., Mandrini, C. H., et al. 2002 Solar Phys., 208, 43

Jacobs, C., Poedts, S., \& van der Holst, B. 2006 A $\mathscr{E} A$, 450, 793

Kliem, B., Török, T. 2006 Phys. Rev. Lett., 96, 255002

Kliem, B., Török, T., \& Thompson, W.T. 2012 Solar Phys., in press

Kliem, B., Rust, S., \& Seehafer, N. 2011 in: A. Bonano, E. de Gouveia Dal Pino, \& A. G. Kosovichev (eds.) Proceedings IAU Symposium No. 274, 125

Kusano, K., Maeshiro, T., Yokoyama, T., \& Sakurai, T. 2002 ApJ, 577, 501

Kusano, K., Yokoyama, T., Maeshiro, T., \& Sakurai, T. 2003 Adv. Space Res., 32, 1931

Kusano, K., Maeshiro, T., Yokoyama, T., \& Sakurai, T. 2004 ApJ, 610, 537

LaBonte, B. J., Georgoulis, M. K., \& Rust, D. M. 2007 ApJ, 671, 955

Lim, E.-K., Jeong, H., Chae, J., \& Moon, Y.-J. 2007 ApJ, 656, 1167

Liu, Y. \& Schuck, P. W. 2012 ApJ, in press

Longcope, D.W. 2004 ApJ, 612, 1181

Low, B. C. 1996 Solar Phys., 167, 217

Low, B. C. \& Zhang, M. 2002 ApJ (Letters), 564, L53

MacNeice, P., Antiochos, S. K., Phillips, A., et al. 2004 ApJ, 614, 1028

Mandrini, C. H., Pohjolainen, S., Dasso, S., et al. 2005 A $\& A$, 434, 725

Moon, Y.-J., Chae, J., Choe, G. S., et al. 2002a ApJ, 574, 1066

Moon, Y.-J., Chae, J., Wang, H., Choe, G. S., \& Park, Y. D. 2002b ApJ, 580, 528

Moore, R. L., Sterling, A. C., Hudson, H. S., \& Lemen J. R. 2001 ApJ, 552, 883

Nindos, A. \& Zhang, H. 2002 ApJ (Letters), 573, L133

Nindos, A., Zhang, J., \& Zhang, H. 2003 ApJ, 594, 1033

Nindos, A. \& Andrews, M. D. 2004 ApJ (Letters), 616, L175

Nindos, A., Patsourakos, S., \& Wiegelmann, T. 2012, ApJ (Letters), 748, L6

November, L. J. \& Simon, G. W. 1988 ApJ, 333, 427

Pariat, E., Démoulin, P., \& Berger, M. A. 2005 A $\& A$, 439, 1191

Pariat, E., Démoulin, P., \& Nindos, A. 2007 Adv. Sp. Res., 39, 1706

Pariat, E., Nindos, A., Démoulin, P., \& Berger, M. A. 2006 A $\mathscr{S} A, 452,623$

Pevtsov, A.A. $2000 \mathrm{ApJ}, 553,560$

Pevtsov, A.A., Canfield, R.C., \& Metcalf, T.R. 1995 ApJ (Letters), 440, L109

Pevtsov, A. A., Canfield, R. C., \& Latushko, S. M. 2001 ApJ (Letters), 549, L261

Phillips, A. D., MacNeice, P. J., \& Antiochos, S. K. 2005 ApJ (Letters), 624, L129

Ravindra, B., Longcope, D. W., \& Abbett, W. P. 2008 ApJ, 677, 751

Rudenko, G. V. \& Myshyakov, I.I. 2011 Solar Phys., 270, 165

Rust, D. M. 1994 Geophys. Res. Lett., 21, 241

Rust, D. M. \& Kumar, A. 1996 ApJ, 464, L199

Schuck, P. W. 2006 ApJ, 646, 1358

Schuck, P. W. 2008 ApJ, 683, 1134

Thalmann, J. K., Inhester, B., \& Wiegelmann, T. 2011 Solar Phys., 272, 243

Tziotziou, K., Georgoulis, M. K. \& Raouafi, N. 2012 ApJ (Letters), 779, L4

Török, T. \& Kliem, B. 2005 ApJ, 630, L97

Valori, G., Démoulin, P., \& Pariat, E. 2012 Solar Phys., 278, 347

van Ballegooijen, A. A. \& Martens P. C. H. 1989 ApJ, 343, 971

Welsch, B. T., Abbett, W. P., DeRosa, M. L., et al. 2007 ApJ, 670, 1434

Welsch, B. T., Fisher, G. H., Abbett, W. P., \& Regnier, S. 2004 ApJ, 610, 1148

Zhang, M. \& Low, B. C. $2001 A p J, 561,406$

Zhang, M. \& Low, B. C. 2003 ApJ, 584, 479

Zhang, M. \& Flyer, N. 2008 ApJ, 683, 1160

Zhang, M., Flyer, N., \& Low, B.C. 2006 ApJ, 644, 575

Zhang, M., Flyer, N., \& Low, B. C. 2012 ApJ, 755, 78

Zhang, Y., Kitai, R., \& Takizawa, K. 2012 ApJ, 751, 85

Zuccarello, F. P., Jacobs, C., Soenen, A., et al. 2009 A\&\&A, 507, 441 